\title{
Composition des principaux tissus adipeux de la chèvre Alpine en fin de lactation
}

\author{
P. BAS ${ }^{(*)}$, Y CHILLIARD ${ }^{(* *)}$, P. MORAND-FEHR ${ }^{(*)}$, \\ Annie ROUZEAU ${ }^{(*)}$, Nadine MANDRAN ${ }^{(*)}$ \\ * INRA, Station de Nutrition et Alimentation, \\ I.N.A. Paris-Grignon \\ 16, rue Claude-Bernard, F 75231 Paris Cedex 05 \\ ** INRA, Laboratoire de Lactation, \\ Centre de Recherches de Clermont-Ferrand-Theix, F 63122 Ceyrat
}

\begin{abstract}
Résumé
Huit chèvres de race Alpine ont été abattues entre la $35^{\mathrm{e}}$ et la $39^{\mathrm{e}}$ semaine de lactation sur lesquelles, 5 tissus adipeux viscéraux : grand épiploon (GE), petit épiploon (PE), périrénal (PR), mésentérique (MES) et péricardique (PC) et 6 tissus adipeux sous-cutanés : inguinal (ING), sternal (ST), caudal (CA), costal (COS), mammaire (MAM) et lombaire (LO) ont été prélevés. Leurs teneurs en eau et en lipides ont été mesurées et leur composition en acides gras a été déterminée.

A ce stade de lactation, le poids total des tissus adipeux viscéraux varie entre 2985 et $7688 \mathrm{~g}$. Leur teneur en eau est comprise entre $5(\mathrm{GE})$ et $12 \%(\mathrm{PC})$. Elle est plus élevée dans les tissus sous-cutanés $(30 \%$ dans $\mathrm{CA})$.

La composition en acides gras présente de grandes variations entre tissus et entre chèvres. Les tissus adipeux viscéraux sont plus riches en acides gras saturés que les tissus sous-cutanés. Selon l'indice de saturation $(14: 0+15: 0+16: 0+17: 0+18: 0)$, les tissus adipeux viscéraux se discriminent dans l'ordre décroissant suivant : $\mathrm{PR}>\mathrm{PC}>\mathrm{MES}>\mathrm{GE}>\mathrm{PE}$ et les tissus souscutanés : ING $>$ MAM $>$ LO $>\mathrm{COS}>\mathrm{CA}>\mathrm{ST}$. Ces tissus sous-cutanés ont par contre des teneurs élevées en acides gras insaturés $(16: 1 \mathrm{n}-7,17: 1 \mathrm{n}-8,18: 1 \mathrm{n}-7$ et surtout 18:1 $\mathrm{n}-9)$ et en acides gras ramifiés saturés. Les corrélations entre les acides gras (16:0, 17:0, 18:0, 16:1 $n-7,17: 1 n-8$ et 18:1 $n-9$ ) sont variables selon les tissus mais leurs évolutions semblent très liées car dans les 11 tissus analysés les rapports 18:0/18:1 $n$ - 9 sont très étroitement corrélés avec les rapports 17:0/17:1 $\mathrm{n}-8(\mathrm{r}>0.9)$ et dans une moindre mesure avec les rapports 16:0/16:1 $\mathrm{n}-7$.
\end{abstract}

Mots clés: Chèvre Alpine, tissu adipeux, eau, lipides, acides gras.

\section{Introduction}

Les lipides des tissus adipeux des femelles à haut potentiel laitier sont mobilisés intensément en début de lactation, ce qui compense leur déficit énergétique et leur permet d'exprimer leur potentiel laitier (ChIlliard, 1985 et 1987). Ces réserves sont ensuite reconstituées en fin de lactation et pendant la période sèche. 
Alors que les variations de poids et de composition (eau, lipides, acides gras) ont été étudiées dans les tissus adipeux des chevreaux de races laitières (SAuvant, Bas \& Morand-Fehr, 1979 ; Bas et al., 1981 ; Bas, Rouzeau \& Morand-Fehr, 1985 et 1986 ; Morand-Fehr et al., 1985 ; Bas et al., 1987), il n'existe que peu de données chez les chèvres adultes (Duncan et al., 1976, Gall, 1980 ; Chilliard, 1985), bien que leur connaissance soit nécessaire pour une meilleure compréhension des aspects quantitatifs et qualitatifs de la mobilisation et de la reconstitution des réserves corporelles.

L'objet de la présente étude a donc été de caractériser les principaux tissus adipeux de la chèvre adulte en fin de lactation. Une partie des résultats a déjà fait l'objet d'une communication préliminaire (ChILliard et al., 1981).

\section{Matériels et méthodes}

Huit chèvres de race Alpine alimentées individuellement et recevant le même régime $(1,1 \mathrm{~kg}$ de foin de luzerne, $300 \mathrm{~g}$ d'aliment concentré par animal plus $300 \mathrm{~g}$ par $\mathrm{kg}$ de lait brut, chaque jour) sont abattues (au mois d'octobre), entre la $35^{\mathrm{c}}$ et la $39^{\mathrm{c}}$ semaine de lactation.

Les tissus adipeux viscéraux : épiploïque grand (GE) et petit épiploon (PE), mésentérique (MES), périrénal (PR) et péricardique $(\mathrm{PC})$ de même que deux tissus sous-cutanés : mammaire (MAM) et sternal externe (ST) sont rapidement prélevés et pesés après l'abattage. Des parties aliquotes de ces tissus ainsi que celles de 4 autres tissus adipeux sous-cutanés : inguinal (ING), caudal, à la base de la queue (CA), costal $(\mathrm{COS})$ (prélevé entre la $7^{\mathrm{e}}$ et la $8^{\mathrm{e}}$ côte à mi-distance entre la colonne vertébrale et le sternum) et lombaire (LO) (au-dessus de la $4^{\mathrm{c}}$ vertèbre lombaire) sont aussitôt congelés à $-50^{\circ} \mathrm{C}$ dans des sachets préalablement tarés.

La teneur en eau est déterminée après 72 heures de lyophilisation et la teneur en lipides par extraction sous reflux pendant 6 heures à l'éther éthylique à partir des échantillons déshydratés puis découpés en lamelles de quelques milligrammes.

Les acides gras de ces extraits sont séparés par chromatographie en phase gazeuse sur une colonne capillaire de verre, après avoir été transbutylés. Ces esters sont obtenus, après 30 minutes de reflux, à partir de $100 \mathrm{mg}$ d'extrait lipidique en présence de $10 \mathrm{ml}$ de butanol chlorhydrique à $3 \%(\mathrm{~V} / \mathrm{V})$ et élimination du butanol en excès par lavage à l'eau. Ils sont séparés sur un chromatographe Girdel ${ }^{(1)}$ de type 75 , muni d'un injecteur évaporateur à aiguille.

La colonne de 50 mètres de longueur environ et de $0,25 \mathrm{~mm}$ de diamètre intérieur est imprégnée de Carbowax $20 \mathrm{M}$ par la méthode dynamique à index de mercure (Prevot \& Mordret, 1976). La température du détecteur à ionisation de flamme est de $250^{\circ} \mathrm{C}$. Le débit du gaz vecteur (hydrogène) est de $2 \mathrm{ml} / \mathrm{mn}$ sous une pression de 0,8 bar tandis que la fuite est réglée à $20 \mathrm{ml} / \mathrm{mn}$. L'analyse est effectuée à la température isotherme de $190^{\circ} \mathrm{C}$. Dans ces conditions, l'efficacité de la colonne mesurée avec l'acide heptadécanoïque est de 60000 plateaux théoriques. Les constituants ainsi séparés sont quantifiés grâce à un intégrateur électronique (Delsi Icap 10.5 ). Leur

(1) Girdel Delsi. 51, rue de Verdun, 92150 Suresnes (France). 
identification est réalisée à partir des longueurs équivalentes de chaîne (LEC) de MıwA et al. (1960) calculées sur des échantillons hydrogénés ou non. Les LEC sont déterminées par interpolation entre 2 acides gras saturés à chaîne linéaire paire, puis comparées soit à celle d'étalons (Sigma) ${ }^{(2)}$ chromatographiés dans des conditions opératoires identiques soit à celles déjà publiées (FLANZY et al., 1976; NeLSON, 1974 ; JAMIESON, 1975 ; LAM \& LIE KEN JIE, 1976a et b). L'hydrogénation des acides gras insaturés est effectuée, à partir de quelques milligrammes (1 à 10) d'esters butyliques en solution dans $8 \mathrm{ml}$ de butanol, sous un léger courant d'hydrogène (pendant au moins 1 heure), en présence de $20 \mathrm{mg}$ de platine oxydé (Touzart \& Matignon) ${ }^{(3)}$ selon une technique dérivée de celle de Poukka, Vasenius \& Turpeinen, (1962).

Dans cette étude, les acides gras saturés, portant une ramification sur la chaîne carbonée, sont répartis en 3 groupes d'après leur ordre d'élution. Les acides à 15 $(15 R), 16(16 R)$ et $17(17 R)$ atomes de carbone représentent la somme des acides qui éluent respectivement entre le 14:0 et le 15:0, entre le 15:0 et le 16:0 et entre le 16:0 et le 17:0.

\section{Résultats}

\section{A. Poids des tissus adipeux (tabl. 1)}

Le poids des tissus adipeux viscéraux (TAV) $(\mathrm{GE}+\mathrm{PE}+\mathrm{MES}+\mathrm{PR}+\mathrm{PC})$ varie selon les chèvres de 2985 à $7688 \mathrm{~g}$ [soit de 6,6 à $14,4 \%$ du poids vif corrigé (poids vif - poids des digesta)]. Le grand épiploon est le tissu le plus développé $(48,8 \%$ des TAV, écart-type $(s)=4,95)$ puis viennent par ordre décroissant le périrénal $(26,4 \%$ des TAV, $\mathrm{s}=2,88)$ le mésentérique $(16,2 \%$ des TAV, $\mathrm{s}=3,26)$ et enfin, le péricardique $(4,4 \%$ des TAV, $s=1,32)$ et le petit épiploon $(4,1 \% \operatorname{des} \mathrm{TAV}, \mathrm{s}=0,75)$.

\section{B. Composition des tissus adipeux (tabl. 1)}

\section{Teneurs en lipides et en eau}

Les tissus adipeux viscéraux présentent des teneurs en eau plus faibles et des teneurs en lipides plus élevées que les tissus adipeux sous-cutanés. Toutefois, les teneurs en eau des tissus viscéraux : PC, MES et PE sont plus élevées que celles des tissus GE et PR, et proches de celles des 2 tissus sous-cutanés LO et ING. La teneur en lipides est corrélée négativement, de façon très étroite, avec la teneur en eau pour l'ensemble des tissus analysés à l'exception du petit épiploon $(\mathrm{r}=0,767 ; \mathrm{P}<0,05$; $\mathrm{n}=8$ ).

(2) Sigma P.O., Box 14508, Saint-Louis, M-63178 (U.S.A.).

(3) Touzart et Matignon, 8, rue Eugène-Hénaff, 94000 Vitry-sur-Seine (France). 


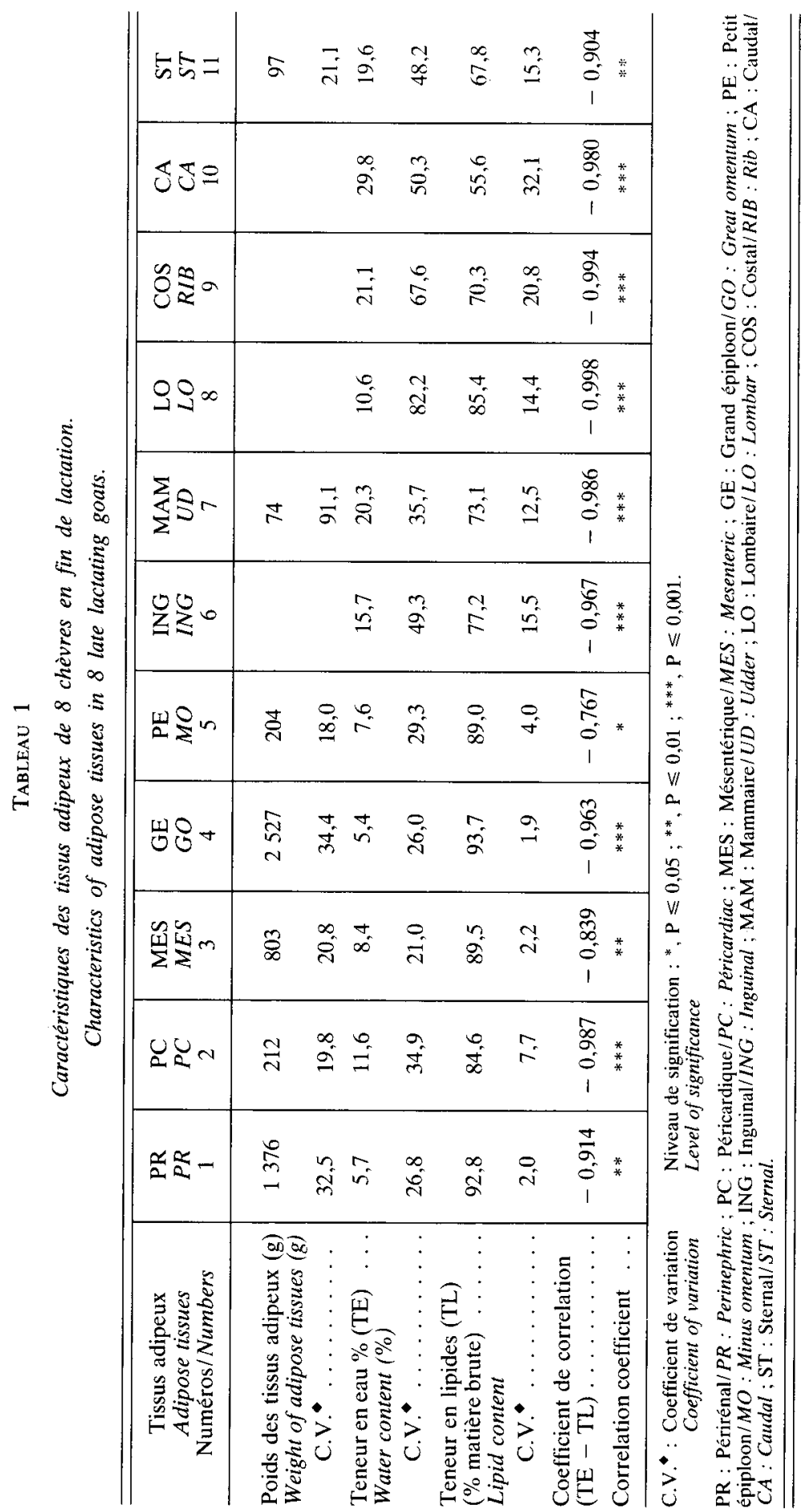




\section{Composition en acides gras des tissus adipeux}

\section{Identification}

Ces tissus adipeux sont surtout constitués d'acides gras à 16 ou 18 atomes de carbone qui représentent de 90 à $91 \%$ (en poids) des acides gras dans les tissus viscéraux et de 86 à $90 \%$ dans le cas des tissus sous-cutanés. Les acides gras monoinsaturés semblent appartenir à 3 familles homologues distinctes. Les acides gras ayant pour LEC 16,17 et 18,17 sont assimilés aux acides $16: 1 \mathrm{n}-9$ et $18: 1 \mathrm{n}-9$, ceux ayant pour LEC 16,25 et 18,25 aux acides $16: 1 \mathrm{n}-7$ et 18:1 $\mathrm{n}-7$ et enfin, la LEC 17,21 est attribuée au 17:1 n - 8. Avec ce type de colonne, les isomères (cis ou trans) ne peuvent être séparés et chaque pic peut correspondre à un mélange cis + trans.

Trois grandes familles d'acides gras saturés ramifiés ont été rencontrées. La plus importante est celle dont les LEC $(13,53,14,53,15,52,16,52$ et 17,51) sont associées aux acides gras iso $14,15,16,17$ et 18 . La seconde famille semble être constituée par les acides antéiso 13,15 et 17 (LEC $12,6714,65$ et 16,66 ) tandis que la $3^{\mathrm{c}}$ pourrait correspondre aux acides gras dont le radical méthyle est porté par le $4^{\mathrm{c}}$ atome de carbone par rapport à la fonction carboxylique (LEC 14,26 15,26 et 16,28) DunCAN, Lough \& GarTon, 1974).

\section{Variations relatives des acides gras (fig. 1)}

A l'exception du 16:0, la variabilité des acides gras est très importante entre tissus, ainsi qu'entre chèvres pour un même tissu.
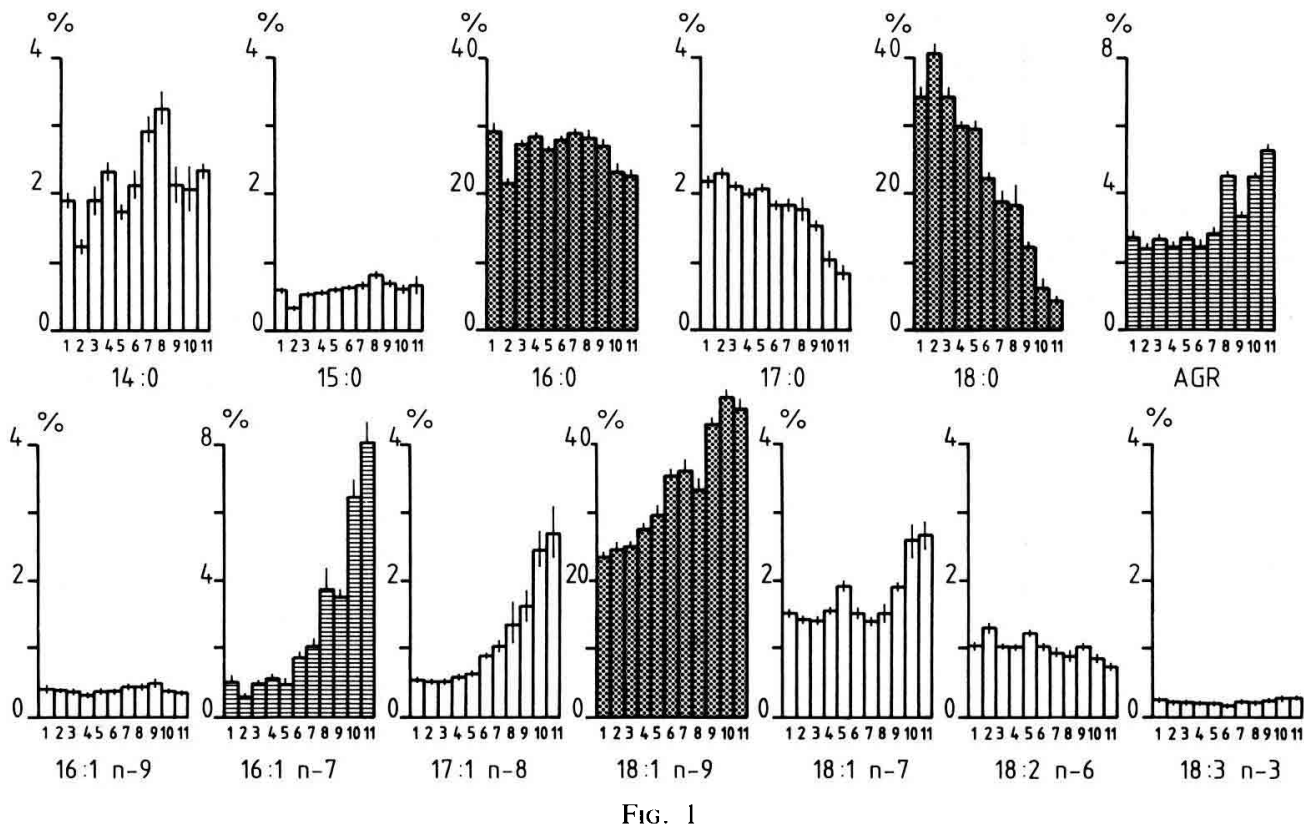

Composition en acides gras de différents tissus adipeux de 8 chèvres en fin de lactation.

Fatty acid composition of adipose tissues in 8 late lactating goats.

Légende (cf. tableau l) tiret = erreur type, hyphen = standard error of the mean. 
L'indice de saturation (IS) défini par la somme des pourcentages des acides gras saturés à chaîne linéaire $(14: 0+15: 0+16: 0+17: 0+18: 0)$ est nettement plus élevé dans les tissus viscéraux que dans les tissus sous-cutanés (fig. 2). L'évolution de cet indice est dû pour une plus grande part au 18:0 et dans une moindre mesure au 17:0 alors qu'au contraire les teneurs en acides gras à chaîne carbonée moyenne (14:0 et 15:0) ont tendance à être plus élevées dans les tissus sous-cutanés que dans les tissus viscéraux. Le 16:0 est le moins variable avec un coefficient de variation intertissus inférieur à $10 \%$. L'indice des acides monoènes (IDM) correspondant à la somme des pourcentages des acides gras $(16: 1+17: 1+18: 1)$ n'est supérieur à l'IS que dans les tissus costal, caudal et sternal (fig. 2). Cet indice, qui ne comprend pas les acides linoléique et linolénique dont les teneurs semblent indépendantes du site anatomique, est inférieur à l'indice de double liaison de 1 à $1,6 \%$.

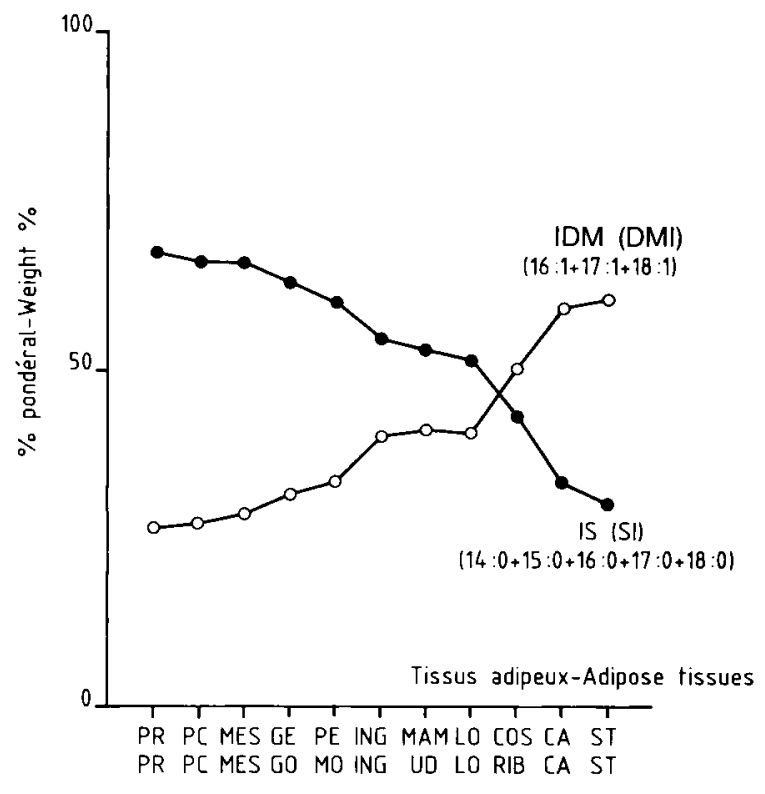

Fig. 2

Indices de saturation (IS) et des acides gras monoènes (IDM) de 11 tissus adipeux de chèvres. Saturation (SI) and monoene acid (DMI) indices in 11 adipose tissues of goats.

Légende : cf. tableau $I$.

Les tissus adipeux viscéraux se classent par ordre de saturation décroissante comme suit : $\mathrm{PR}>\mathrm{PC}>\mathrm{MES}>\mathrm{GE}>\mathrm{PE}$ et les tissus sous-cutanés dans l'ordre suivant : $\mathrm{ING}>\mathrm{MAM}>\mathrm{LO}>\mathrm{COS}>\mathrm{CA}>\mathrm{ST}$. Le tissu péricardique plus riche en 18:0 que le tissu périrénal, a un IS plus faible à cause de taux plus faibles de 14:0, 15:0 et 16:0. Les tissus adipeux viscéraux possèdent moins de 18:1 $\mathrm{n}-9$, de 17:1 n-8, de 16:1 $\mathrm{n}-7$ et d'acides gras ramifiés, à 15 et 17 atomes de carbone en particulier (non représentés sur la figure 1) que les tissus sous-cutanés. Les autres acides gras dépendent moins du site anatomique. 
Les tissus sous-cutanés (sternal et costal) les plus riches en acides gras monoènes $(16: 1 \mathrm{n}-7,17: 1 \mathrm{n}-8,18: 1 \mathrm{n}-7$ et 18:1 $\mathrm{n}-9)$ présentent toutefois les teneurs les plus faibles de 18:2 $n-6$. Les tissus adipeux inguinal et mammaire ont des compositions intermédiaires entre celles des autres tissus sous-cutanés et celles des tissus viscéraux. Le tissu adipeux lombaire se distingue par des taux élevés de 14:0, 15:0 et aussi d'acides gras ramifiés (surtout 16R).

Les liaisons intratissus entre les principaux acides gras sont indiquées dans les figures $3 a$ et $3 b$. Les corrélations entre les acides $16: 1 n-7$ et $17: 1 n-8$ sont significatives au seuil de $5 \%$ dans tous les tissus excepté dans les tissus mésentérique, costal et sternal, où elles ne le sont qu'au seuil de $10 \%$ (fig. 3a). Les acides 17:1 $n-8$ et 18:1 n -9 ne sont corrélés positivement de façon significative $(P<5 \%$ ) que dans les tissus viscéraux et le tissu sous-cutané lombaire alors que dans les autres tissus sous-cutanés, la liaison est, ou moins étroite (ING, MAM, COS, CA P:NS) ou négative (ST : $r=-0,762 \mathrm{P}<5 \%$ ). Les variations du 17:0 suivent dans l'ensemble celles du 18:1, mais de façon significative $(\mathrm{P}<5 \%)$ seulement dans les tissus MES, PE, ING, LO, CA et ST. Par contre, elles semblent le plus souvent indépendantes des variations du 16:0 (PC, PE, MAM, LO, COS, CA) ou leur sont opposées dans les autres tissus.

Les liaisons entre les acides gras saturés et les acides monoènes de même nombre d'atomes de carbone sont très différentes selon les acides et les sites. Seul le 18:1 n - 9 semble corrélé négativement au 18:0 (P<5\% dans PR, PC, GO, PE, ING, MAM et LO et $\mathrm{P}<10 \%$ dans COS) (fig. 3b).
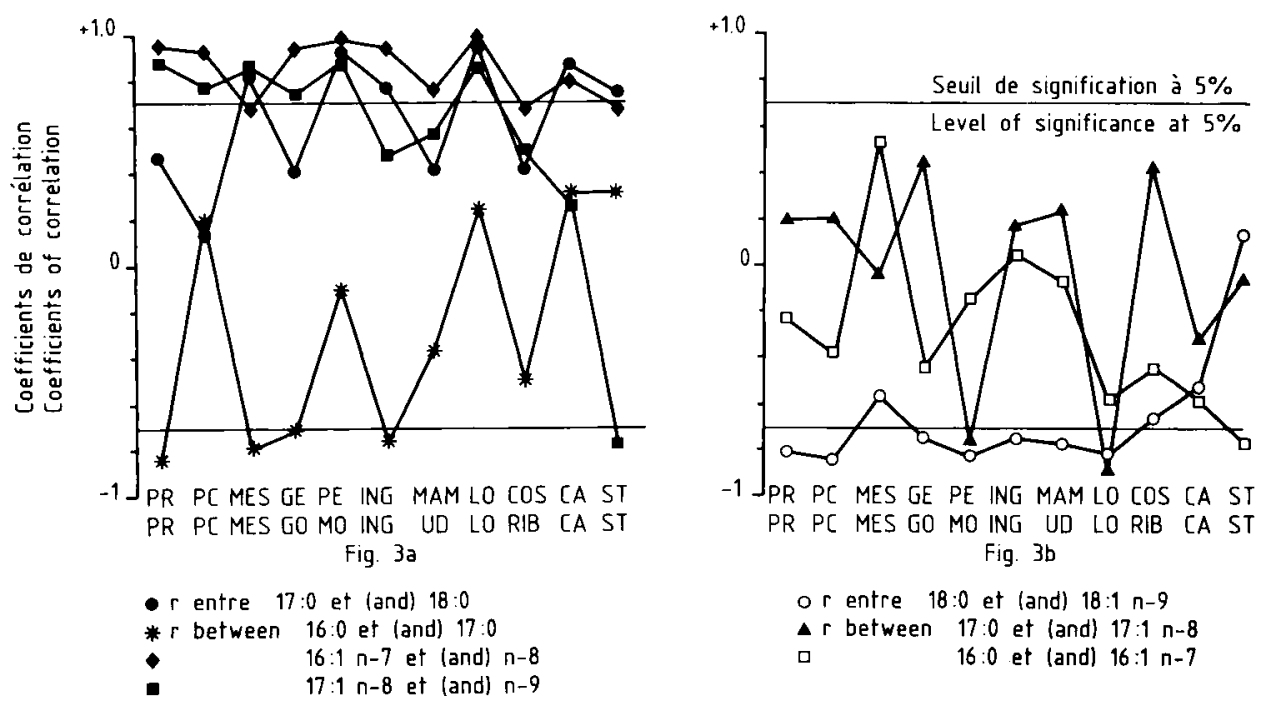

Fig. 3

Coefficients de corrélation entre les pourcentages de quelques acides gras dans les tissus adipeux de 8 chèvres en fin de lactation.

Coefficients of correlation between fatty acids in 8 late lactating goats. 
Les acides 16:0, 16:1 $\mathrm{n}-7,17: 0,17: 1 \mathrm{n}-8,18: 0$ et 18:1 $\mathrm{n}-9$ ne sont que rarement corrélés 2 à 2 . Leurs variations semblent néanmoins liées car le rapport 18:0/18:1 n - est corrélé positivement, d'une manière hautement significative dans tous les tissus, avec le rapport 17:0/17:1 n - 8, et de façon moins étroite avec celui 16:0/16:1 $n-7$. Par contre, il est quasiment indépendant (ou lié négativement dans LO) du rapport 16:0/16:1 $\mathrm{n}-9$ (fig. 4).

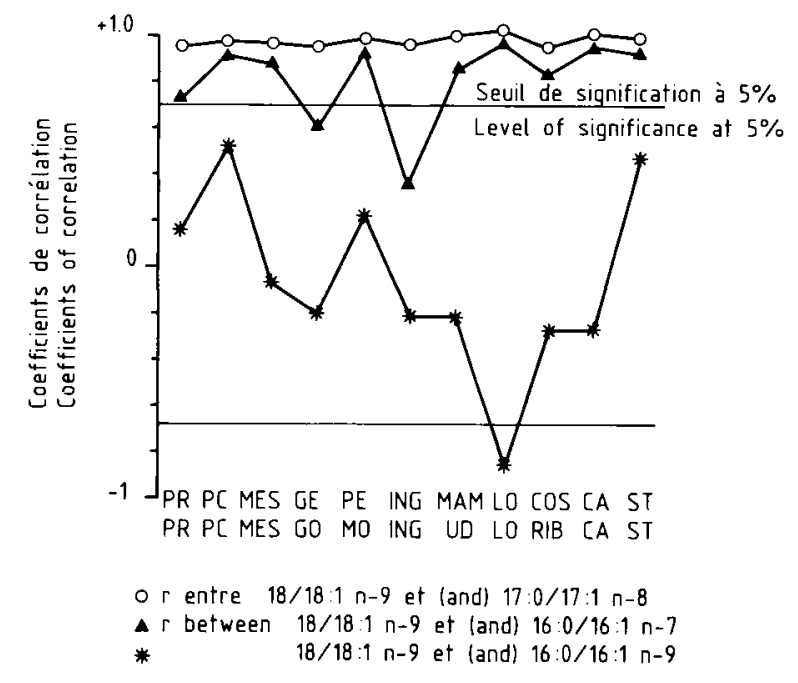

FIG. 4

Coefficients de corrélation entre les rapports "acides gras saturés/acides gras monoènes correspondants".

Coefficients of correlation between ratios of saturated to monoene fatty acids.

Les acides gras ramifiés totaux représentent de 2,5 à $2,8 \%$ de l'ensemble des acides gras dans les tissus viscéraux. Leurs pourcentages sont plus élevés dans les tissus sous-cutanés caudal, lombaire et surtout sternal (fig. 1). Les groupes d'acides gras ramifiés sont corrélés positivement entre eux dans tous les tissus, mais plus étroitement dans le tissu ST $(\mathrm{P}<1 \%)$.

Dans ce tissu, les taux élevés d'acides gras ramifiés chez 2 chèvres sont dus principalement aux acides dont les LEC sont 14,26, 15,26 et 16,28. L'élévation du pourcentage de l'ensemble des acides gras ramifiés est accompagnée par celle des acides $14,0,15,0$ et $17,1 \mathrm{n}-8(\mathrm{r}=+0,864 \mathrm{P}<1 \%, \mathrm{r}=+0,979 \mathrm{P}<1 \%$ et $\mathrm{r}=+0,908$ $\mathrm{P}<1 \% \mathrm{n}=8$, respectivement entre les acides 14:0, 15:0 et 17,1 $\mathrm{n}=8$ et les acides gras ramifiés totaux). Par contre, le $18: 1$ n -9 est corrélé négativement avec la somme des acides gras ramifiés $(r=0,915 \mathrm{P}<1 \%)$. 


\section{Discussion}

Les chèvres Alpines en fin de lactation présentent un développement important des tissus adipeux viscéraux alors que leurs dépôts sous-cutanés sont quantitativement peu développés. Les plus fortes teneurs en lipides des dépôts adipeux viscéraux soulignent aussi leur rôle pour l'accumulation des réserves énergétiques à une période de la lactation où la lipolyse est très réduite par rapport à la lipogenèse (CHILliard, 1985).

Le fort développement des dépôts adipeux viscéraux pourrait être lié au caractère laitier des chèvres Alpines, en accord avec les observations de Gall et al. (1972). En effet, chez les bovins, le pourcentage de dépôts viscéraux dans les dépôts adipeux totaux est plus élevé chez les Holstein que dans les races à viande (Charolais ou Hereford) (Callow, 1961 ; Beranger \& Robelin, 1977 ; Robelin 1978 et 1986a ; Jones \& Mac Leod, 1981 ; Kempster, 1981 ; Truscott, Wood et Mac Fie, 1983 ; Wright et Russel, 1984 ; Chilliard et Robelin, 1985). Par contre, le faible développement des dépôts sous-cutanés est peut-être plutôt une caractéristique de certaines races caprines, puisque chez les bovins, les races laitières présentent un pourcentage de dépôts adipeux sous-cutanés dans les tissus adipeux totaux qụi est intermédiaire entre celui des races à viande maigres (Charolais) et grasses (Hereford) (revue de Robelin, 1986b).

La composition en acides gras des tissus adipeux de la chèvre est proche de celle des autres ruminants. Bien qu'elle résulte à la fois d'une lipogenèse de novo, du prélèvement des acides gras des triglycérides sanguins et de leur élongation et de leur désaturation, elle semble surtout influencée par l'activité métabolique du rumen. En plus de leur synthèse tissulaire de novo, les acides palmitique, stéarique et oléique proviennent de la digestion des acides gras d'origine alimentaire et leurs dérivés hydrogénés, ainsi que des lipides synthétisés par les bactéries et les protozoaires. La biohydrogénation par les hydrogénases microbiennes des acides gras polyinsaturés du régime (Hawke \& Silcock, 1970; Noble, Moore \& Harfoot, 1974) est en grande partie responsable de la pauvreté de ces tissus en acides linoléique et linolénique et de leur richesse en acides stéarique et palmitique, de même que de la présence en quantité non négligeable d'acides spécifiques tels que le 18:1 $\mathrm{n}-7$. Cet acide en tant qu'intermédiaire des étapes d'hydrogénation, serait probablement en grande partie sous forme trans dont l'incorporation dans les tissus adipeux de ruminants a été observée notamment par Dawson \& Kemp, 1970 et par Steele, Noble \& Moore, 1971. Les lipides des bactéries et des protozoaires contiennent en outre des acides gras impairs et des acides gras ramifiés iso et antéiso susceptibles d'être digérés, absorbés puis prélevés par les tissus (Hansen, 1966; Williams \& Dinusson, 1973; Emmanuel, 1978). Ces acides impairs sont synthétisés dans le rumen à partir du malonyl $\mathrm{CoA}$ et du propionyl $\mathrm{CoA}$ provenant de l'acide propionique et d'acides aminés tels que la méthionine, la thréonine et la valine. Dans les acides gras iso ou antéiso, l'élément de départ de la synthèse est soit de l'isobutyryl CoA, de l'isovaleryl CoA ou du méthyl butyryl CoA. Dans ces conditions, la valine, la leucine et l'isoleucine conduisent respectivement soit à des acides gras iso pairs, soit iso impairs ou soit à des antéiso impairs. Cet apport d'acides gras ramifiés d'origine microbienne explique leur présence à de faibles taux dans tous les tissus mais non leurs taux particulièrement élevés dans les tissus adipeux souscutanés lombaire, caudal et sternal. Dans ces derniers tissus, l'élévation du taux d'acides ramifiés serait due en grande partie aux acides gras dont la ramification est plus proche du groupement carboxyle. 
Dans les tissus sous-cutanés, les taux plus élevés d'acides gras impairs et d'acides gras ramifiés pourraient aussi résulter d'une synthèse de novo tissulaire soit à partir de propionate ou de son dérivé le méthylmalonyl CoA puisque lorsqu'il est en excès par rapport aux possibilités de prélèvement par le foie, le propionate peut se substituer à l'acétate pour la synthèse des acides gras. Ces taux plus élevés d'acides gras impairs et ramifiés dans les tissus sous-cutanés que dans les tissus viscéraux ont souvent été observés chez les agneaux (Duncan, Orskov \& Garton, 1974) et les chevreaux (Duncan, Orskov \& Garton, 1976 ; Sauvant, Bas \& Morand-Fehr, 1979 ; Bas et al., 1981 ; Castellanos, Ruelas et al., 1982 ; Bas et al., 1987) avec des rations riches en aliments concentrés ou avec des céréales telles que l'orge. Cette caractéristique, de même que l'absence de $14: 1$ et de $15: 1$ et les taux relativement réduits de 16:1 semblent indiquer que, de ce point de vue, le métabolisme lipidique de la chèvre est plus proche de celui des ovins que des bovins (Hartman \& Shortland, 1961 ; Duncan \& Garton, 1967 ; Hubbard \& Pocklinton, 1968 ; Christie \& Moore, 1971 ; Marmer, Maxwell et Williams, 1984).

Les pourcentages plus élevés des acides monoènes $(16: 1 \mathrm{n}-7,17: 1 \mathrm{n}-8$ et 18:1 $n-9)$ dans les tissus adipeux sous-cutanés que dans les tissus viscéraux pourraient être dus à une plus grande activité de désaturation dans les premiers (Whale \& Garton, 1972).

Les liaisons très étroites qui lient les acides gras $(16: 0,17: 0,18: 0,16: 1 \mathrm{n}-7,17: 1$ $\mathrm{n}-8, \quad 18: 1 \mathrm{n}-9)$ permettent de penser que l'activité de désaturation régule les pourcentages respectifs de ces acides. Mais le fait que les rapports 18:0/18:1 n -9 soient plus étroitement corrélés avec les rapports 17:0/17:1 $\mathrm{n}-8$ qu'avec les rapports 16:0/16:1 $\mathrm{n}-7$ peut être attribué à une élongation d'une partie du 16:1 $\mathrm{n}-7$ en 18:1 $\mathrm{n}-7$ (de forme cis) et dans une moindre mesure à l'activité de désaturation légèrement moins active vis-à-vis du 16:0 que du 17:0 (PAulsrud et al., 1970).

En outre, les taux plus élevés de 18:1 n - 7 dans les tissus sous-cutanés sternal et caudal dont l'activité LPL est faible (ChILliaRD et al., 1981) conduisent à penser que ce constituant est un mélange de formes cis et trans et que la forme cis proviendrait de l'élongation du 16:1 n -7 .

Les écarts entre les teneurs en eau et entre les indices de saturation des tissus adipeux viscéraux d'une part et sous-cutanés d'autre part induisent, en partie, les différences de fermeté de ces 2 groupes de tissus. L'indice de saturation diminuant à mesure que les tissus sont plus exposés aux températures extérieures (Cramer \& Marchello, 1964 ; L'Estrange \& Mulvihill, 1975), la localisation des tissus adipeux sous-cutanés : inguinal, lombaire et mammaire explique leurs indices de saturation plus élevés que ceux des autres tissus sous-cutanés (costal, caudal et sternal). En revanche, les compositions des tissus viscéraux (péricardique, périrénal, mésentérique, grand et petit épiploon) semblent moins influencées par la température. Cependant, les tissus les plus saturés (périrénal et péricardique) sont les plus internes mais d'autres facteurs tels que la nature des acides gras prélevés et l'activité métabolique des tissus adipeux pourraient aussi intervenir.

\section{Conclusion}

Cette étude a permis de préciser de façon détaillée la composition en acides gras des tissus adipeux de 11 sites anatomiques de la chèvre adulte en fin de lactation. 
Comme chez les autres ruminants, cette composition est fortement influencée par l'activité du rumen. Toutefois l'absence de 14:1, de 15:1, les taux réduits de 16:1 et les taux élevés d'acides gras ramifiés semblent rapprocher plus les caprins des ovins que des bovins.

Les tissus adipeux sous-cutanés sont plus riches en eau et en acides gras monoinsaturés et à chaîne ramifiée que les tissus viscéraux. Il existe toutefois de grandes différences entre les tissus des différents sites anatomiques à l'intérieur de ces deux grands groupes de tissus adipeux, peut-être en liaison avec le degré d'exposition aux températures extérieures.

Les liaisons très étroites qui existent entre les rapports 18:0/18:1 n -9 et 17:0/17:1 n -8 dans tous les tissus suggèrent que ceux-ci résultent de l'action d'une même $\Delta 9$ désaturase, dont l'activité est variable selon les individus et selon les sites anatomiques.

Reçu en octobre 1986.

Accepté en novembre 1987.

\section{Summary \\ Composition of adipose tissue in Alpine goats during late lactation}

Eight Alpine goats were slaughtered between the 35th and 39th week of lactation.

Five visceral adipose tissues (AT) : great omentum (GO), minus omentum (MO), perinephric (PR), mesenteric (MES) and pericardic (PC) and 6 subcutaneous AT: inguinal (ING), sternal (ST), caudal (CA) rib (RIB), udder (UD) and loin (LO) were studied for their water and lipid content and for fatty acid composition by GLC with glass capillary column.

Visceral AT weights fluctuated from 2985 to $7688 \mathrm{~g}$ (table 1). GO was the heavier. AT water content was comprised between 5 to $30 \%$. GO had the lowest water content and the caudal the highest. Lipid content was always highly negatively correlated with water content.

High branched chain fatty acid percentage in subcutaneous AT and lack of C14:1 and C15:1 were characteristic of goats. And by this way their metabolism was nearer to those of sheep than bovine (fig. 1).

Fatty acid composition was highly variable between tissues and between goats. Visceral AT were more saturated than subcutaneous AT. In visceral AT saturation indices C14:0 + C15:0 + $\mathrm{C} 16: 0+\mathrm{C} 17: 0+\mathrm{C} 18: 0$ ) decreased in the following way: $\mathrm{PR}>\mathrm{PC}>\mathrm{MES}>\mathrm{GO}>\mathrm{MO}$ (fig. 2). Yet PC was the richest in C18:0. CA and ST had few C18:0, C17:0 and C18:2 $n-6$ but a lot of $\mathrm{C} 16: 1 \mathrm{n}-7, \mathrm{C} 17: 1 \mathrm{n}-8, \mathrm{C} 18: 1 \mathrm{n}-7, \mathrm{C} 18: 1 \mathrm{n}-9$ and branched chain fatty acids.

ING and UD had intermediate composition between internal and other subcutaneous AT.

C17:1 $n-8$ was positively correlated with $\mathrm{C} 16: 1 \mathrm{n}-7$ in most At but with C18:1 $\mathbf{n}-9$, only in visceral and lombar AT. C17:0 had often similar variations than C18:0 but their coefficient of correlation wer not so high as between $\mathrm{C} 17: 1 \mathrm{n}-8$ and $\mathrm{C} 18: 1 \mathrm{n}-9$ and on the contrary C17:0 seemed to be independent of C16:0 in subcutaneous AT or negatively correlated in PR, MES and GO. Yet, $\mathrm{C} 17: 0, \mathrm{C17:1} \mathrm{n}-8, \mathrm{C} 18: 0$ and $\mathrm{C} 18: 1 \mathrm{n}-9$ were always indirectly linked because in each adipose tissue $\mathrm{C} 18: 0 / \mathrm{C} 18: 1 \mathrm{n}-9$ was highly correlated to $\mathrm{C} 17: 0 / \mathrm{C} 17: 1 \mathrm{n}-8(\mathrm{r}>0.9)$. with C16:0/C16:1 $\mathrm{n}-7$, the coefficients of correlation were smaller.

Key Words : Alpine Goat, adipose tissue, water, lipid, fatty acid composition. 


\section{Références bibliographiques}

Bas P., Hervieu J., Morand-Fehr P., Sauvant D., 1981. Facteurs influençant la composition des graisses chez le chevreau de boucherie. Incidence sur la qualité des gras de carcasses. In "Nutrition and systems of goat feeding". International Symposium Tours (France 12-15 mai 1981) ed. by Morand-Fehr P., Bourbouze A. \& de Simiane M. INRA-ITOVIC. Vol. I, p. 90100.

Bas P., Rouzeau A., Morand-Fehr P., 1985. Poids et métabolisme des réserves lipidiques au cours de la croissance du chevreau. Reprod. Nutr. Dévelop., 25, 275-285.

Bas P., Rouzeau A., Morand-Fehr P., 1986. Lipogenèse des tissus adipeux de chevreaux sevrés à 4,6 et 8 semaines. Reprod. Nutr. Dévelop., 26, 649-658.

Bas P., Morand-Fehr P., Rouzeau A., 1987. Evolution de la composition des tissus adipeux du chevreau mâle sevré à 4,6 ou 8 semaines. Reprod. Nutr. Dévelop., 27, 313-314.

Beranger C., Robelin J., 1977. Influence du mode d'élevage, de la sélection et de l'alimentation sur l'état d'engraissement des bovins. Ann. Biol. anim. Biochim. Biophys., 6, 905-922.

Callow E.H., 1961. Comparative studies of meat. VII, A comparison between Hereford, Dairy short-horn and Friesan steers on four levels of nutrition. J. agric. Sci., Camb., 56, 265-282.

Castellanos Ruelas A., Bas P., Sauvant D., Morand-Fehr P., 1982. Influencia de la alimentacion des acido propionico y de la castration sobre el creciumendo des cabrito y de la composicion en acidos grasos doe los depositos. Technica Pecuaria en Mexico, diciembre, Suppl. 9, 13-19.

Chilliard Y., Sauvant D., Bas P., Pascal G., Morand-Fehr P., 1981. Importance relative et activités métaboliques des différents tissus adipeux de la chèvre laitière. In "Nutrition and systems of goat feeding ". International Symposium Tours (France 12-15 mai 1981) ed. by Morand-Fehr P., Bourbouze A., de Simiane A. INRA-ITOVIC Vol. I, p. 80-89.

ChILliard Y., 1985. Métabolisme du tissu adipeux, lipogenèse mammaire et activités lipoprotéinelipasique chez la chèvre au cours du cycle gestation-lactation (134 pp. 1323 réf.). Thèse Doct. Etat ès-Sci., Univ. Paris 6 (France).

ChIlliard Y., 1987. Revue bibliographique: Variations quantitatives et métabolisme des lipides dans les tissus adipeux et le foie au cours du cycle gestation-lactation. II. Chez la brebis et la vache. Reprod. Nutr. Dévelop., 27, 327-398.

Chilliard Y., Robelin J., 1985. Activité lipoprotéine lipasique de différents dépôts adipeux et ses relations avec la taille des adipocytes chez la vache tarie en cours d'engraissement, ou en début de lactation. Reprod. Nutr. Dévelop., 25, 287-294.

Christie W.W., Moore J.H., 1971. Structure of triglycerides isolated from various sheep tissues. $J$. Sci. Fd. Agric., 22, 120-124.

Cramer D.A., Marchello J.A., 1964. Seasonal and sex patterns in fat composition of lambs. $J$. anim. Sci., 23, 1002-1010.

Dawson R.M.C., KemP P., 1970. Biohydrogenation of dietary fats in ruminants. In "Physiology of digestion and metabolism in the ruminant" ed. by Phillipson A.T., Oriel Press, England, pp. 504-518.

DunCan W.R.H., Garton G.A., 1967. The fatty acid composition and intramolecular structure of triglycerides derived from different sites in the body of the sheep. J. Sci. Fd. Agric., 18, 99102 .

Duncan W.R.H., Lough A.K., Garton G.A., 1974. Characterisation of branched chain fatty acids from subcutaneous triacylglycerols of barley-fed lambs. Lipids, 9, 669-673.

Duncan W.R.H., Orskov E.R., Garton G.A., 1974. Effect of different dietary cereals on the occurrence of branched-chain fatty acids in lambs fat. Prod. Nutr. Soc., 33, 81A-82A.

Duncan W.R.H., Orskov E.R., Garton G.A., 1976. Fatty acid composition of triglycerides of goat fed on a barley-rich diet. Prod. Nutr. Soc., 35, 89A-90A.

Emmanuel B., 1978. The relative contribution of propionate and long chain even fatty acids to the production of long-chain and odd-numbered fatty acids in rumen bacteria. Biochem. Biophys. Acta, 528, 239-246. 
Flanzy J., Boudon M, Leger C, Pihet J., 1976. Application of Carbowax 20M on open-tubular liquid phase in analyses of nutritionnally important fat and oils. J. Chromatog. Sci., 14, 17-24.

Gall C., 1980. Relationship between body conformation and production in dairy goats. $J$. Dairy Sci., 63, 1768-1781.

Gall C., Frahm K., Graf F., Osterkorn K., 1972. Body conformation and milk production in dairy goats. I. Estimation of total body fat and total muscle weight by part dissection data. $Z$. Tierzuecht. Z.uechtbiol., 89, 123-128.

HANSEN R.P., 1966. Phytanic acid and other branched-chain fatty acid constituents of bovine rumen bacteria. J. Dairy Res., 33, 151-155.

Hartman J.L., Shortland F.B., 1961. Fatty acid composition and other characteristics of subcutaneous fat from New Zealand oxen and sheep. New Zealand J. Sci., 4, 16-25.

HAWKE J.C., SILCOCK W.R., 1970. The in vitro rates of lipolysis and biohydrogenation in rumen contents. Biochem. Biophys. Acta, 218, 201-212.

Hubbard A.W., Pocklingron W.D., 1968. Distribution of fatty acids in lipids as an aid to the identification of animal tissue. I. Bovine, porcine, ovine and some avian species. J. Sci. Fd. Agric., 19, 571-577.

JAMIESON G.R., 1975. GLC identification techniques for long-chain unsaturated fatty acids. $J$. Chromatog. Sci., 13, 491-497.

Jones S.D.M., Mac Leod G.K., 1981. The feedlot performance and carcass composition of young and mature cull Holsteins cows. Can. J. anim. Sci., 61, 593-599.

Kempster A.J., 1981. Fat partition and distribution in the carcass of cattle, sheep and pigs. Meat Sci., 5, 83-98.

LAM C.H., LIE KEN JIE M.S.F., 1976a. The gas liquid chromatographic properties of all dimethylene interrupted methyl cis, cis octadecadienoates. J. Chromatog., 117, 365-374.

LAM C.H., LIE KEN JIE M.S.F., 1976b. The gas liquid chromatographic properties of all dimethylene interrupted methyl trans, trans octadecadienoates. J. Chromatog., 121, 303-311.

L'Estrange J.L., Mulvihill T.A., 1975. A survey of fat characteristics of lamb with particular reference to the soft fat condition in intensively fed lambs. J. agric., Sci., Camb., 84, 281-290.

Marmer W.N., Maxwell R.J., Williams J.E., 1984. Effects of dietary regimen and tissue site on bovine fatty acid profiles. J. anim. Sci., 59, 109-121.

Miwa T.K., Mikolajczak K.L., Earle F.R., WolfF I.A., 1960. Gas chromatographic characterization of fatty acids. Identification constants for mono and dicarboxylic methyl esters. Anal. Chem., 32, 1739-1742.

Morand-Fehr P., Bas P., Rouzeau A., Hervieu J., 1985. Development and characteristics of adipose deposits in male kids during growth from birth to weaning. Anim. Prod., 41, 349-357.

Nelson G.J., 1974. Elution characteristics of fatty acids methyl esters on capillary columns. Lipids, 9, 254-263.

Noble R.C., Moore J.H., Harfoot C.G., 1974. Observations on the pattern on biohydrogenation of esterified and unesterified linoleic acid in the rumen. Br. J. Nutr., 31, 99-108.

PoukKa R., Vasenius L., Turpeinen O., 1962. Catalytic hydrogenation of fatty acid methyl esters for gas liquid chromatography. J. Lipid Res., 3, 128-129.

Paulsrud J.R., Stewart S.E., GrafF G., Holman R.T., 1970. Desaturation of saturated fatty acids by rat liver microsomes. Lipids, 5, 611-616.

Prevot A.F., Mordret F.X., 1976. Utilisation des colonnes capillaires de verre pour l'analyse des corps gras par chromatographie en phase gazeuse. Rev. Fse Corps Gras, 23, 409-423.

Robelin J., 1978. Répartition des dépôts adipeux chez les bovins selon l'état d'engraissement, le sexe et la race. Bull. Techn. CRZV Theix, INRA, 34, 31-34.

Robelin J., 1986a. Composition corporelle des bovins : Evolution au cours du développement et différences entre races. Thèse de doctorat ès Sciences, Univ. Clermont-Ferrand II, 391 p.

RobeliN J., 1986b. Growth of adipose tissues in cattle : partitioning between deposit, chemical composition and cellularity. A review. Livest. prod. Sci., 14, 349-364.

Sauvant D., Bas P., Morand-Fehr P., 1979. Production de chevreaux lourds : II. Influence du niveau d'ingestion de lait et du sevrage sur les performances et la composition du tissu adipeux. Ann. Zootech., 28, 73-92. 
Steele W., Noble R.C., Moore J.H., 1971. The effect of dietary soybean oil on milk fat composition in the cow. J. dairy Res., 38, 49-55.

Truscott T.G., Wood J.D., Macfie H.J.H., 1983. Fat deposition in Hereford and Friesian steers. 1. Body composition and partitioning of fat between depots. J. agric. Sci., 100, 257-270.

Whale K.W.J., Garton G.A., 1972. Desaturation of stearic acid by sheep tissue microsomes. Proc. Nutr. Soc., 31, 110A-111A.

Williams P.P., Dinusson W.E., 1973. Amino acid and fatty acid composition of bovine ruminal bacteria and protozoa. J. anim. Sci., 36, 151-155.

Wright I.A., Russel A.J.F., 1984. Partition of fat, body composition and body condition score in mature cows. Anim. Prod., 38, 23-32. 